Check for updates

Cite this: RSC Adv., 2017, 7, 30250

\title{
Paclitaxel-loaded pluronic F127/P123 silica nanocapsules with surface conjugated rhTRAIL for targeted cancer therapy
}

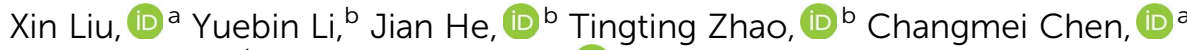 \\ Haoshuang $\mathrm{Gu}^{\mathrm{b}}$ and Xingguo Wang (D) *a
}

A novel anticancer drug PFPSNT, paclitaxel (PTX)-loaded polymeric F127/P123 silica nanocapsules conjugated with TRAIL (tumor necrosis factor (TNF)-related apoptosis-inducing ligand), was designed and synthesized. Transmission electron microscopy (TEM), dynamic light scattering (DLS), UV-vis absorption and particle size analysis revealed that the synthesized PFPSNT was a spherical particle with a size of $24 \mathrm{~nm}$ and excellent colloidal stability. Drug loading efficiency (DL\%) and encapsulation ratio (ER\%) were $0.39 \%$ and $26.5 \%$, and its cumulative release profile was linear. In vitro study showed that PFPSNT had marked cytotoxic and apoptotic activities towards HepG2 (liver cancer cell line) and CaSki (cervical cell line) cells with the $\mathrm{IC}_{50}$ values of $921.07 \mathrm{ng} \mathrm{mL} \mathrm{L}^{-1}$ and $236.24 \mathrm{ng} \mathrm{mL} \mathrm{L}^{-1}$, respectively. The antitumor efficacy of PFPSNT was evaluated by using a HepG2-xenografted BALB/c nu/nu mice model. In vivo study showed PFPSNT markedly inhibited HepG2 hepatocellular carcinoma, and its antitumor efficacy was more superior than those of TRAIL, PTX or PTX/F127/P123 silica nanocapsules. Our studies provided a novel strategy to synthesize anticancer nanodrugs with mutiple functions, and demonstrated that PFPSNT was a very effective anticancer drug. This novel nanodrug should be a promising drug for targeted cancer therapy to treat complex cancers.

Received 21st April 2017 Accepted 5th June 2017

DOI: $10.1039 / c 7 r a 04503 d$

rsc.li/rsc-advances that the combination of chemotherapy drugs and TRAIL can significantly enhance the apoptosis of tumor cells and reduce the dose of chemotherapy drugs. TRAIL combined with doxorubicin indeed displayed strong inhibitions for a wide variety of tumors. ${ }^{13,14}$ Paclitaxel (PTX), a cytoskeletal drug targeting tubulin, is commonly used in clinical therapy. It is usually used as an inhibitor to inhibit tumor cell proliferation through stabilizing microtubule network and inhibiting microtubule dynamics. ${ }^{15}$ It has been reported that PTX can be used as a sensitizing agent towards TRAIL. ${ }^{16-19}$ However, PTX, as a chemotherapeutic drug, has poor aqueous solubility and low therapeutic index..$^{20}$ In clinical therapy, PTX is used as a mixture solution composed of $50 \%(\mathrm{v} / \mathrm{v})$ dehydrated alcohol and $50 \%(\mathrm{v} /$ v) cremophor EL (polyoxyl 35 castor oil) with 5-20-fold dilution with saline or dextrose solution before administration. ${ }^{21}$ Like other chemotherapeutic drugs, its usage causes several side effects, such as hair loss, muscle and joint pains, and diarrhea. ${ }^{22}$ Improving PTX solubility and reduce its side effects are certainly required. Recently, Xin H. et al. ${ }^{23}$ has found that the embedment of PTX in nanocapsules can significantly improve PTX solubility.

Nanocapsules are made up of a nontoxic polymeric membrane and an inner liquid core at the nanoscale level. ${ }^{24}$ Nanocapsules used as vesicular systems for cancer treatment have a myriad of benefits, including the increase of drug dissolution rate, drug delivery, controllable release and precise 
targeting. ${ }^{25,26}$ Up to today, several amphiphilic organic molecules including lipids, block copolymers and polypeptides ${ }^{27-29}$ have been used to assemble micelles to encapsulate hydrophobic anticancer drugs, such as paclitaxel, doxorubicin and cisplatin. ${ }^{30-32}$ Silica nanocapsules (SNCs) combine the merits of silica (e.g. mechanical robustness, biocompatibility and great versatility) and capsular nanostructure (e.g. large inner cavity, low density and favourable colloidal properties). SNCs attract people's great attention due to its small size with good colloidal stability, silica shell providing a pathway for chemical drug molecules to diffuse out of the cavity and its onion-like structure favoring loading capacity and release rate of biomedical drugs. ${ }^{33}$ To make nanodrugs more specific for targeting cancer therapy, linking nanocapsules with a delivery target for cancer therapy becomes a hotspot of current research. ${ }^{34}$

More recently, the biodistribution experiment has demonstrated the preferential accumulation of ${ }^{125}$ I-rhTRAIL in CasKixenografted tumors, indicating that TRAIL can also be used as a delivery target vector. ${ }^{35}$ Thus, it is possible for us to link chemotherapeutic drugs, silica nanocapsules and TRAIL together to generate a novel anticancer drug with mutifunctions. The synthesized drugs are predicted to increase dissolution rate, controllable release, co-delivery of chemotherapeutic drugs with TRAIL, precise targeting and robust anticancer activity. In this study, we developed a novel strategy to construct an anticancer targeting drug by using rhTRAIL as a delivery target vector. At first, PTX was embedded inside the pluronic F127/P123 silica nanocapsules, and the then PTXloaded nanocapsules were conjugated with rhTRAIL to form the PTX/F127/P123 silica nanocapsules conjugated with rhTRAIL (PFPSNT). The size and morphology of the PFPSNT, loading parameters for PTX embedment in the nanocapsules and PTX release from the nanocapsules were also examined. Finally, cytotoxic and apoptotic activities as well as the efficacy of inhibiting tumor growth of the PFPSNT were respectively evaluated by using two cancer cell lines HepG2 and CaSki and the HepG2-xenografted $n u / n u$ mouse. Our studies show that the PFPSNT indeed offers a promise of a novel anti-cancer drug with strong tumor-killing efficacy and sustained release of PTX.

\section{Materials and methods}

\subsection{Materials and reagents}

Pluronic F127 $\left(\mathrm{EO}_{106} \mathrm{PO}_{70} \mathrm{EO}_{106}, \mathrm{MW}=12600 \mathrm{~g} \mathrm{~mol}^{-1}\right)$ and $\mathrm{P} 123\left(\mathrm{EO}_{20} \mathrm{PO}_{70} \mathrm{EO}_{20}, \mathrm{MW}=5800 \mathrm{~g} \mathrm{~mol}^{-1}\right)$ were purchased from Sigma Aldrich Co. LLC. The rhTRAIL protein (ab157018) was purchased from Abcam Trading (Shanghai) Company Ltd. Paclitaxel (PTX), N-hydroxysuccinimide (NHS), 1-(3dimethylaminopropyl)-3-ethylcarbodiimide hydrochloride (EDC), acetonitrile, fluorescein diacetate (FDA), tetramethoxysilane (TMOS), pyrene were purchased from Aladdin Industrial Corporation. Chloroform, tetrahydrofuran (THF), methanol, ethanol, xylene, hydrogen peroxide were purchased from Sinopharm Chemical Reagent Co. Ltd. Dimethyl sulfoxide (DMSO) was purchased from Biosharp. The purity of all reagents purchased was $\geq 98 \%$. Phosphate-buffered saline (PBS) and Dulbecco's modified Eagle's medium (DMEM) were purchased from Hyclone. Trypsin-EDTA (0.25\%) was purchased from Life Technologies. Fetal bovine serum (FBS) was purchased from Zhejiang Tianhang Biological Technology Co., Ltd. DAPI dihydrochloride and penicillin-streptomycin $(100 \times)$ was purchased from Beyotime Technologies. Poly formaldehyde (4\%) was purchased from Boster Biological Technology. HepG2 cell line was obtained as a gift from College of Life Sciences, Wuhan University, and CaSki cell line were purchased from FineTest Biotech Co., Ltd.

\subsection{Carboxylation of pluronic F127}

Based on the protocol reported previously ${ }^{36}$ a modified method was used to carboxylate pluronic F127. Briefly, $3.15 \mathrm{~g}$ pluronic F127 was dissolved in the mixture of $13 \mathrm{~mL}$ anhydrous toluene and $0.7 \mathrm{~mL}$ pyridine with heating and stirring in a container filled with nitroge. The final concentration of F127 was $0.25 \mathrm{mM}$. When the temperature approached to $70{ }^{\circ} \mathrm{C}, 75 \mathrm{mg}$ succinic anhydride (SA) or $73.5 \mathrm{mg}$ maleic anhydride (MA) was added. The mixture was continuously heated and stirred. Then, the reflux condensation of the mixture was performed at $90{ }^{\circ} \mathrm{C}$ for $1-5 \mathrm{~h}$. Finally, the distillated residue was washed with chilly ether, and then the synthesized product $\mathrm{F} 127-\mathrm{COOH}$ was dried by vacuuming at $40{ }^{\circ} \mathrm{C}$ for $24 \mathrm{~h}$. Succinic anhydride was chosen to be used for carboxylation of pluronic F127 in the following experiments.

\subsection{Preparation of PTX/F127/P123 silica nanocapsules}

PTX/F127/P123 silica nanocapsules (PTX-NCs) were prepared by using a modified method based on the thin-film hydration ${ }^{37}$ and the addition of tetramethoxysilane (TMOS) $)^{38}{ }^{39}$. Briefly, the carboxylated F127 (90 mg) and pluronic P123 (180 mg) were mixed and dissolved in $1 \mathrm{~mL}$ chloroform, and then sonicated for 15 minutes at room temperature. $4 \mathrm{mg}$ of PTX was added into the F127/P123 solution, followed by sonication for another 15 minutes to form a highly transparent solution. The PTX/F127/ P123 solution was evaporated in a drying oven at $75{ }^{\circ} \mathrm{C}$ under the vacuum state. Then, the resultant thin film was hydrated with $4 \mathrm{~mL}$ water by sonication to obtain a micelle solution. $35 \mu \mathrm{L}$ TMOS, dissolved in $0.5 \mathrm{~mL}$ THF, was taken out and dropped into the micelle solution with stirring at $1000 \mathrm{rpm}$, and then the mixed solution was continuously stirred for 3 days so that THF was evaporation and TMOS was completely hydrolyzed to form the silica shell. Finally, the prepared PTX-NCs were filtrated through $0.22 \mu \mathrm{m}$ filter membrane to remove those unincorporated drug aggregate. All experimental steps were performed at room temperature unless otherwise stated. F127/P123 silica nanocapsules (NCs) without PTX and PTX loaded F127/P123 nanomicelle (PTX-NMs) without addition of TMOS were also made respectively under the similar conditions.

\subsection{Characterization of PTX-NCs}

The morphology of the PTX-NCs was observed by transmission electron microscope (TEM, Tecnai $\mathrm{G}^{2} 20,200 \mathrm{kV}$ ). The size and size distribution of PTX-NCs and PTX-NMs were measured by dynamic light scattering (DLS) (Malvern Zetasizer Nano-S). All measurements were conducted in a glass cuvette and used water as the dispersant. Colloidal stability was also determined 
by DLS. The aqueous suspension of the PTX-NCs and PTX-NMs was diluted for 100-1000 times with distilled water and stirred at room temperature for 4 hours before DLS measurements. UV/ visible absorption spectra were recorded on a UV-3600 spectrophotometer (Shimadzu).

\subsection{Loading efficiency and release of PTX}

The loading parameters for PTX were measured at $229 \mathrm{~nm}$ on an UV-visible spectrophotometer. Drug loaded efficient (DL\%) and encapsulation ratio (ER\%) were calculated according to the following formula as reported by Zhang et al. ${ }^{\mathbf{4 0}}$

DL $(\%)=$ (weight of the PTX embedded in nanocapsules $) /$ (total weight of the PTX plus nanocapsules) $\times 100$

ER $(\%)=$ (weight of the PTX embedded in nanocapsules $) /$ (weight of the feeding PTX) $\times 100$

PTX release behaviors of PTX-NCs, PTX-NMs and PSPFNT were measured by HPLC (Dionex UltiMate 3000 PumP) using the dialysis method as reported by Andersen et al. ${ }^{41}$ Briefly, $3 \mathrm{~mL}$ PTX-NCs was loaded into a dialysis tubing with the molecularweight cutoff (MWCO) of $8000 \mathrm{~g} \mathrm{~mol}^{-1}$, and then dialysized in $30 \mathrm{~mL}$ PBS containing $0.1 \%$ Tween $80(\mathrm{pH}=7.4) .{ }^{42}$ An aliquot of $0.2 \mathrm{~mL}$ was taken out at $0.5,1,2,4,6,8,10$ and $12 \mathrm{~h}$. Meanwhile, the equal amount of release medium $(0.2 \mathrm{~mL})$ was supplemented at each time point to keep total volume unchanged. The content of PTX was estimated by HPLC. Meanwhile, PTX release from stock solution was also conducted under the same condition as a positive control.

\subsection{Surface conjugation of PTX-NCs with TRAIL}

The carboxylic groups of the carboxylated F127 on the surface of PTX-NCs were conjugated with the amino groups of TRAIL proteins to form PFPSNT under the catalysis of EDC and NHS. ${ }^{43}$ Briefly, EDC ( $5 \mathrm{mg})$, NHS (6 mg) and $1 \mathrm{~mL}$ PTX-NCs were mixed in $3 \mathrm{~mL}$ of PBS ( $\mathrm{pH}=6.4$ ), followed by shaking for $1 \mathrm{~h}$ to active the carboxylic group of the F127. The reaction mixture were dialysized in $2 \mathrm{~L} \mathrm{PBS}(\mathrm{pH}=6.4)$ for $12 \mathrm{~h}$ to remove the superfluous EDC and NHS in case self ligation occurred between carboxylic groups and amino groups of TRAIL proteins. Then, 1 mL of the TRAIL with a his-tag at its C-terminal $\left(25 \mu \mathrm{g} \mathrm{mL}^{-1}\right)$ was added, and the mixture was shaken for $24 \mathrm{~h}$ at $4{ }^{\circ} \mathrm{C}$. The Niaffinity chromatography was used to collected the PFPSNT and remove those uncrosslinked nanocapsules. In this experiment, a fluorescent dye pyrene, instead of PTX, was embedded into the F127/P123 silica nanocapsules to estimate conjugation efficiency of the nanocapsules with TRAIL. After Ni-affinity chromatography, the unbound and bound nanocapsules were measured at $338 \mathrm{~nm}$ which is the characteristic absorbance for pyrene. Binding capacity was calculated by the eqn (3).

Binding capacity $(\%)=$ bound nanocapsules/

(bound plus unbound nanocapsules) $\times 100$

\subsection{Cytotoxicity assessment and cellular uptakes of TRAIL- pyrene-NCs}

Cytotoxicity assay was performed by using Cell Counting Kit-8 (CCK-8, Dojindo Molecular Technologies, Inc) to assess the cytotoxicity of TRAIL, TRAIL-NCs, PTX, PFPSNT and PTX-NCs, in which HepG2 and CaSki cell lines were used respectively. Both HepG2 and CaSki cells were cultured in the DMEM medium containing $10 \%$ (v/v) fetal bovine serum (FBS) and 1\% (v/v) penicillin/streptomycin at $37{ }^{\circ} \mathrm{C}$ with $5 \% \mathrm{CO}_{2}$. Briefly, cells were inoculated into a 96 well plates (LAB-TEK, Chambered Coverglass System) at a density of 8000 cells per well. After preincubation overnight, the media were replaced with the fresh DMEM, and then TRAIL, TRAIL-NCs, PTX, PTX-NMs, PTX-NCs and PFPSNT were respectively added to the well at final concentrations of $0-100 \mu \mathrm{g} \mathrm{mL}{ }^{-1}$. After 24 hour incubation, 10 $\mu \mathrm{L}$ CCK-8 solution was added to each well, followed by incubating another $4 \mathrm{~h}$. The optical absorbance for each well was measured at $450 \mathrm{~nm}$ on a Benchmark Plus microplate spectrophotometer (Bio-RAD). The absorbance of each experimental group was calculated by using the value measured in the experimental group minus that of the blank control containing only DMEM medium. All results were again normalized with the positive control group in which cells and the medium were used without additing any nanocapsules or drugs. The date presented in this study was the mean calculated from three independent experiments.

Cellular uptakes of TRAIL-pyrene-NCs by HepG2 cells were also examined using fluorescence microscopy. Briefly, HepG2 cells were plated onto the sterile coverslip in a 6-well plate (LABTEK, Chambered Coverglass System). Cell adensity was $1 \times 10^{6}$ cells per well. After incubation for $8 \mathrm{~h}$, the medium was replaced with fresh medium containing TRAIL-pyrene-NCs or pyreneNCs at final concentrations of $2 \mu \mathrm{g} \mathrm{mL}{ }^{-1}$, and cells were continually incubated for $24 \mathrm{~h}$. All cells in each well were stained by FDA for 15 minutes, and then washed with PBS three times. The coverslip were taken out and cells were observed under a fluorescence microscope (Olympus Fluoview 1000). FDA fluorescent image was acquired at $\lambda_{\mathrm{ex}}=494 \mathrm{~nm}$ and $\lambda_{\mathrm{em}}=$ $520 \mathrm{~nm}$, while the pyrene fluorescent image was acquired at $\lambda_{\mathrm{ex}}$ $=336 \mathrm{~nm}$ and $\lambda_{\mathrm{em}}=384 \mathrm{~nm}$.

\subsection{PFPSNT therapy of HepG2 xenografts}

All animal studies were carried out under the guidelines of the laboratory animal center in Tongji Medical College, Huazhong University of Science and Technology. 4 week-old female athymic BALB/c nu/nu nude mice were purchased from Beijing HFK Bioscience Co., Ltd. The average weight of nude mouse was $15 \mathrm{~g}$. HepG2 cells were harvested and mixed with $80 \%$ matrigel, and then injected into the right flank of each mouse. Inoculum dose for each mouse was $3 \times 10^{6}$ cells by injecting a total volume of $100 \mu \mathrm{L}$. When tumor diameter approached to $0.4-0.6 \mathrm{~cm}$, the nude mice bearing HepG2 xenograft were randomized into five groups (5 mice in each group). $0.35 \mathrm{mg} \mathrm{kg}^{-1}$ TRAIL (7 $\mu \mathrm{g}$ per mouse), $3.5 \mathrm{mg} \mathrm{kg}^{-1}$ PTX (70 $\mu \mathrm{g}$ per mouse), PTX-NCs $(70 \mu \mathrm{g}$ PTX per mouse), $100 \mu \mathrm{L}$ PFPSNT ( $7 \mu \mathrm{g}$ TRAIL and $70 \mu \mathrm{g}$ PTX per mouse); or $100 \mu \mathrm{L}$ PBS (as control) were respectively used to 
treat the nude mice via intraperitoneal (IP) injection. The tumor size was routinely measured, and the tumor volume was calculated according to the eqn (4). The relative tumor volumes were calculated as $V / V_{0}\left(V\right.$ : the tumor volume after treatment $V_{0}$ : the tumor volume before treatment). After 14 day treatment, all mice were sacrificed.

$$
\text { Tumor volume }(V)=\left[(\text { tumor length }) \times(\text { tumor width })^{2}\right] / 2
$$

The anti-tumor efficiency was also evaluated by histological examination. The tumors were taken out from the mice and fixed in $4 \%$ paraformaldehyde solution. The fixed tumors were embedded with paraffin and sectioned into thin pieces, and then stained with Hematoxylin and Eosin staining (H\&E staining), ${ }^{44}$ DAPI $\left(4^{\prime}, 6\right.$-diamidino-2-phenylindole $)$ staining $^{45}$ and TUNEL (terminal deoxynucleotidyl transferase dUTP nick end labeling $)^{\mathbf{4 6}}$ were respectively performed by using the in situ cell death detection kit (Roche, Applied Science). Alterations of tumor tissues and cells at histopathological and apoptosis levels were observed under a light microscope and a confocal laser scanning microscope (Olympus, CX-21). Major organs including heart, liver, spleen, lung and kidney in each group were also collected and examined.

\subsection{Statistical analysis}

Date are presented as mean \pm SDs. Statistical significances were determined using the two way ANOVA. ${ }^{47} P$-values of $<0.05$ were considered statistically significant.

\section{Results and discussion}

\subsection{Characterization of PTX-NCs}

It was reported that PTX/F127/P123 nanomicelles were able to be fabricated by using the thin-film hydration method if pluronic F127 and P123 were mixed with PTX. ${ }^{48}$ The authors found that F127, P123 and PTX was completely dissolvable in a common water-miscible organic solvent (e.g. chloroform), and pluronic F127/P123 in the molten state was an excellent solvent for water-insoluble PTX. However, the resultant thin film was easily hydrated with water by sonication. Recently, another research group found that the hydrophobic PPO (polypropylene oxide) segments and hydrophilic PEO (polyethylene oxide) segments of PPO-PEO block copolymers could rapidly form a core and shell of the micelles. When TMOS was added, it entered the juncture of PEO and PPO, and then hydrolyzed to form silicon dioxide $\left(\mathrm{SiO}_{2}\right)$. Finally, $\mathrm{SiO}_{2}$ deposited between the core and shell of the micelles to form a thin silica layer. This novel type of nanocapsules with a silica layer was more stabile and soluble in aqueous phase. ${ }^{38,49}$ To obtain multifunctional anti-cancer drug with low toxicity, controllable release and precise targeting, pluronic F127 and P123 were used to make the PTX-loaded F127/P123 silica nanocapsules by combining two methods as mentioned above. The reason for choosing F127 and P123 was that the hydrophilic PEO segment of F127 was longer than that of P123. A long hydrophilic PEO segment might enhance the solubility of the synthesized nanocapsules, whereas a short PEO segment of P123 might benefit PTXloading capacity in the nanocapsule.

In order to crosslink TRAIL with the PTX-NCs, pluronic F127 was initially carboxylated, and then the F127-COOH was used to replace F127 during the preparation of PTX-NCs. Fig. 1B shows the FTIR spectra of F127 and carboxylated F127. The stretching vibration of hydroxyl group of $F 127$ at $1630 \mathrm{~cm}^{-1}$ disappeared in the carboxylated F127, and the latter emerged a novel peak at $1735 \mathrm{~cm}^{-1}$ attributing to its carbonyl group. The analysis of FTIR spectra indicated that the pluronic F127 indeed was carboxylated successfully to form the F127-COOH. Its carboxylation efficiency, determined by conductometric titration, reached up to $96.5 \%$ or $92.8 \%$, when succinic anhydride or maleic anhydride was used.

The morphology and size of the PTX-NCs were examined. The synthesized PTX-NCs were observed by transmission electron microscopy (TEM), and their morphology and sizes were shown in Fig. 2A. As shown in Fig. 2A, the PTX-NCs indeed exhibited a typical structure of silica nanocapsules. The dark silica shell displayed a significantly high electron density, and its thickness measured by the software nanomeasure was $5.28 \mathrm{~nm}$. The bright core was composed of PPO blocks of F127 and P123 containing PTX. The particle size measured by the TEM images was $12.98 \mathrm{~nm}$. As shown in Fig. 2A, the nanocapsules appeared to link each other. This might perhaps result from the short PEO chains of P123 unable to provide enough steric hindrances to keep the silica nanocapsules from agglomerating together. Pluronic P123/F127 mixed polymeric nanocapsules appeared aggregation was reported by $\mathrm{Hsu} \mathrm{B}$. Y. W. et al. ${ }^{50}$ In addition, the nanocapsules also showed obvious Tyndell effects when a laser was used to illuminate them (see the inset in Fig. 2A).

The size of the PTX-NCs was determined by using DLS, and the results were summarized in Fig. 2B. The PTX-NCs showed a relatively narrow peak of size distribution with a low polydispersity index (PDI), similar to that for the PTX-NMs. However, the average diameter for the PTX-NCs was close to $24 \mathrm{~nm}$ with an acceptable PDI of 0.217 . By contrast, the average diameter for the PTX-NMs was $21 \mathrm{~nm}$ with a PDI value of 0.122 . The size of $24 \mathrm{~nm}$ obtained from the DLS measurement was larger than that from the TEM image. The explanation for size discrepancy could be that the particle size measured by DLS includes the extension of free PEO chains into water, but TEM images do not.

Colloidal stability is another important index of nanocapsules. Polymeric micelles will gradual disintegrate when they are diluted below the critical micelle concentration (CMC). ${ }^{51}$ By contrast, silica nanocapsules would avoid of disintegration due to their silica-shell formation. The colloidal stability of the PTX-NCs and the PTX-NMs were compared. After dilutition of 100-1000 folds with $\mathrm{dH}_{2} \mathrm{O}$, DLS assays were performed, and the results were summarized in Fig. $2 \mathrm{C}$ and D. As shown in Fig. 2C, the size distributions of PTX-NCs were almost unchanged even if it was diluted to 1000 folds. Different from the PTX-NCs, the PTX-NMs displayed a marked change in its size distributions when its dilution was in excess of 200 folds. When the dilution approached to 500 or 1000 folds, several new 

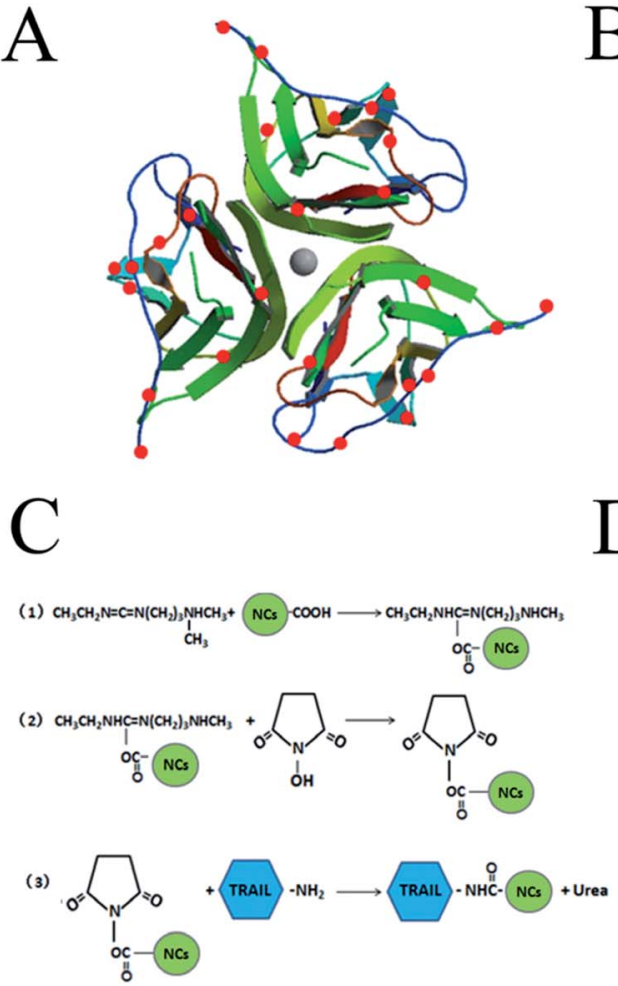

$\mathrm{B}$

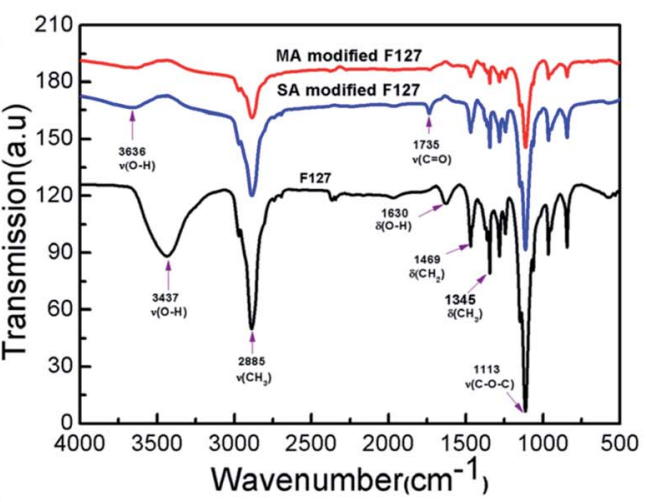

$\mathrm{D}$

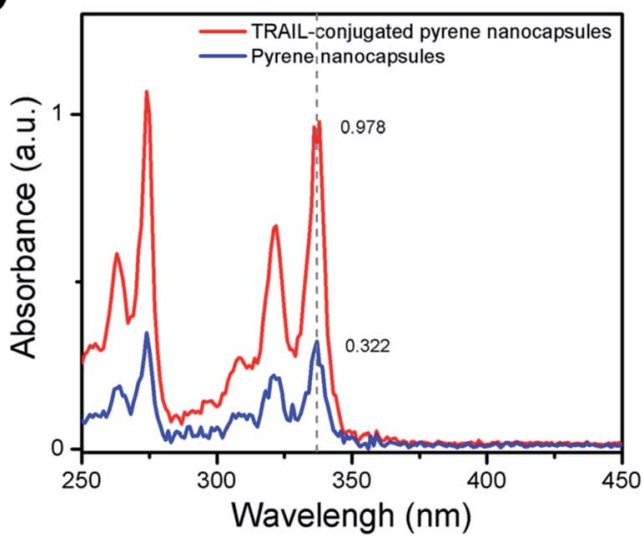

Fig. 1 (A) A molecular model presenting amino groups on the surface of TRAIL analyzed by PyMol images; (B) FTIR spectra of carboxylated-F127 block copolymers; (C) a schematic describing the reaction process of the conjugation of PTX-NCs with TRAIL; (D) wavelength scanning of TRAILconjugated pyrene/F127/P123 silica nanocapsules and pyrene/F127/P123 silica nanocapsules.

peaks appeared. In addition, the hydrodynamic sizes of the PTX-NMs also showed a gradual increase at 100-200 fold dilutions. The appearance of several new peaks implied that the PTX-NMs were easily disintegrated under the dilution conditions, and the embedded PTX might easily be exposed to water solvent. These results in Fig. 2C and D clearly demonstrated that the PTX-NCs were more stable than the PTX-NMs.

\subsection{Loading capacity and release of PTX in PTX-NCs}

The PTX-loading parameters for both PTX-NCs and PTX-NMs were examined by measuring the PTX absorption at $229 \mathrm{~nm}$. The results were summarized in Table 1 . The effective concentrations of PTX in the PTX-NCs and the PTX-NMs were $265.4 \mu \mathrm{g}$ $\mathrm{mL}^{-1}$ and $852.3 \mu \mathrm{g} \mathrm{mL} \mathrm{m}^{-1}$, which were much larger than $0.3 \mu \mathrm{g}$ $\mathrm{mL}^{-1}$ for PTX intrinsic water solubility. ${ }^{22}$ More than 800 -fold increase of PTX-loading concentration in either nanocapsules or micelles illustrated that the microencapsulation of PTX markedly improved its aqueous solubility. As shown in Table 1, DL and ER values were given $0.39 \%$ and $26.5 \%$ for the PTX-NCs and $1.26 \%$ and $85.2 \%$ for the PTX-NMs. Low DL and ER values for the PTX-NCs might result from the formation of silica shell when TMOS entered into the core of micelles. Such physical alteration might affect the package of PTX in silica nanocapsules. Although DL and ER values of the PTX-NCs were less than those for the PTX-NMs, the silica shell depositing on the interface between hydrophobic PPO core and hydrophilic PEO chains could prevent drug release directly from the hydrophobic core.

The in vitro cumulative release profiles of PTX for the PTXNMs, PTX-NCs and PFPSNT were shown in Fig. 3. PTX release from stock solution was also investigated as a positive control. It was found that more than $70 \%$ PTX in the stock solution were released in the first $2 \mathrm{~h}$. This suggested that PTX could freely diffuse through the dialysis membrane. As shown in Fig. 3, a hyperbolic curve for PTX cumulative release profile indicated that PTX release from the PTX-NMs was quite rapid at early stage. More than $30 \%$ of PTX in the PTX-NMs was released at $30 \mathrm{~min}$, and nearly $56 \%$ PTX was released at $2 \mathrm{~h}$. By contrast, the PTX-NCs released only $2.3 \%$ and $10.4 \%$ of PTX at the same time points. Even at 12 hours, the amount of PTX released from PTXNCs was less than $62 \%$. More importantly, the cumulative release profile of PTX was almost linear in this case, suggesting that PTX release was slow and steady during the testing process. These results clearly demonstrated that the PTX-NCs indeed sustained the release of PTX. Such a gradual release of PTX could benefit anticancer therapy because the sustained release of PTX from silica nanocapsules would decrease PTX concentration in blood. Less PTX in blood reduced the nonspecific toxicity of PTX to normal cells. In addition, both PFPSNT and PTX-NCs showed similar profiles of PTX cumulative release. No 
A

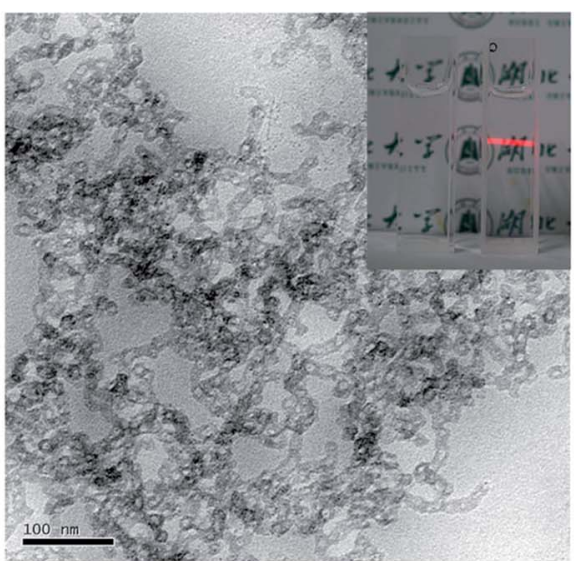

C

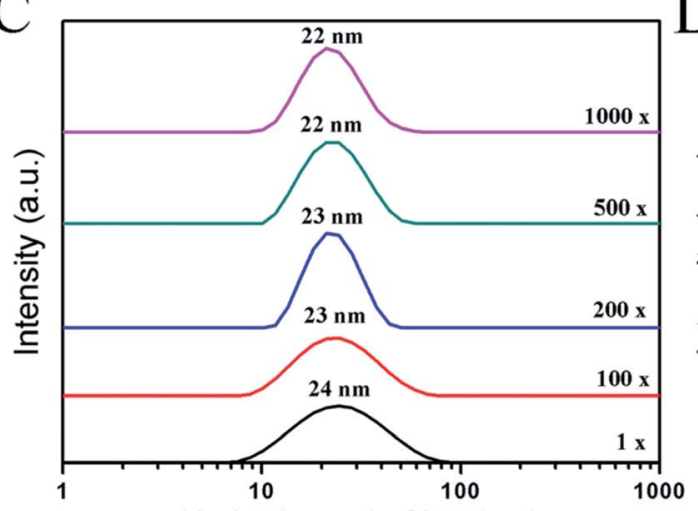

Hydrodynamic Size $(\mathrm{nm})$
B

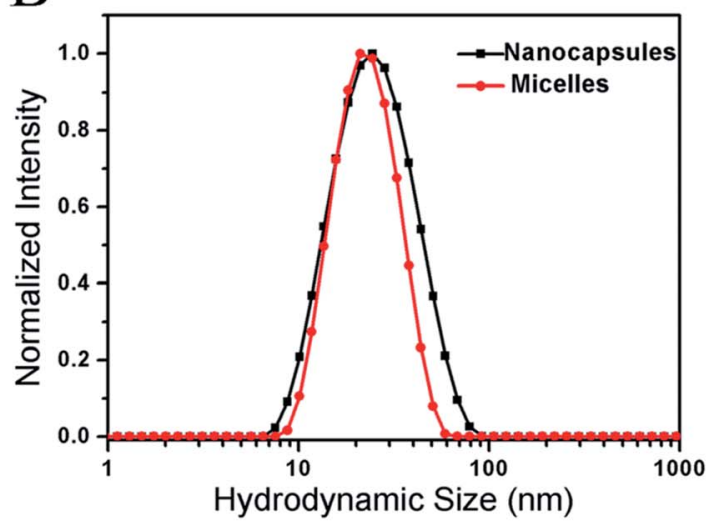

$\mathrm{D}$

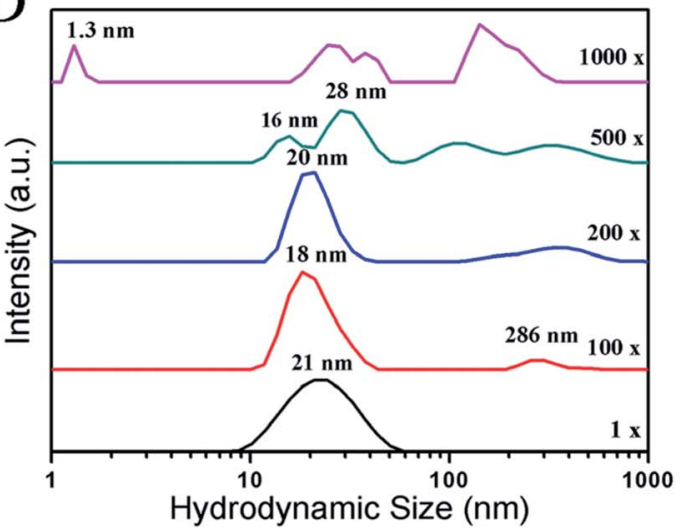

Fig. 2 Characterization of PTX-NCs and PTX-NMs. (A) TEM images of PTX-NCs; (B) the size determination of PTX-NCs and PTX-NMs by DLS; DLS tests of colloidal stability for PTX-NCs (C) and PTX-NMs (D).

Table 1 Comparison of PTX-loaded capacities in PTX-NMs and PTX$\mathrm{NCs}^{a}$

\begin{tabular}{llll}
\hline & & & $\begin{array}{l}\text { PTX concentration } \\
\text { in nanoparticles }\left(\mu \mathrm{gL}^{-1}\right)\end{array}$ \\
& DL $(\%)$ & ER $(\%)$ & \\
PTX-NMs & 1.26 & $85.2 \%$ & 852.3 \\
PTX-NCs & 0.39 & $26.5 \%$ & 265.4
\end{tabular}

${ }^{a}$ DL: drug loaded efficient; ER: encapsulation ratio.

significant difference between release profiles of PFPSNT and PTX-NCs indicated that TRAIL conjugation did not affected the release behavior of PTX from silica nanocapsules.

\subsection{Conjugation efficiency of PTX-NCs and TRAIL}

The carboxylic groups of the carboxylated F127 on the surface of PTX-NCs were conjugated with the amino groups of TRAIL to form a PTX/F127/P123 silica nanocapsule-TRAIL (PFPSNT). 3D image of the surface amino groups of TRAIL was analysed by using PyMol software ${ }^{52}$ and shown in Fig. 1A. The TRAIL protein, used in this study, contains 168 amino acids, in which only 10 lysine and arginine residues with a free amino group exist. The analysis by PyMol software found that only 9 amino groups were exposed on the surface of each TRAIL subunit.
Thus, a trimeric TRAIL, acting as an active form, contains 27 amino groups on its surface. Fig. 1C illustrated the reaction scheme of TRAIL conjugation with PTX-NCs. Firstly, EDC activated the carboxylic group of the nanocapsules to form an $o$ acylisoureaester intermediate. Then NHS reacted with the $o$ acylisoureaester intermediate to form $N$-hydroxysuccinimideester. $N$-Hydroxysuccinimide-ester of nanocapsules was stable,

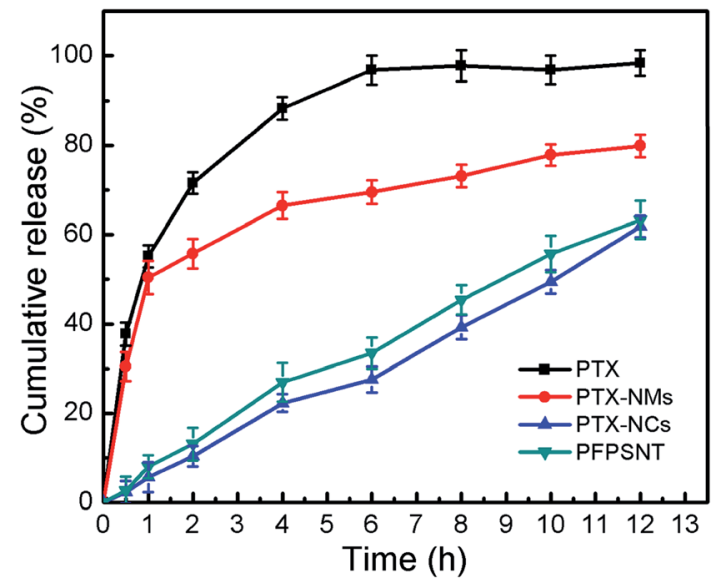

Fig. 3 Cumulative release of PTX from PTX-NMs, PTX-NCs and PFPSNT. 
and actively reacted with the amino group of TRAIL. Finally, TRAIL-conjugated silica nanocapsules were obtained.

To determine the conjugation efficiency, a fluorescent dye, pyrene, was loaded into the F127/P123 silica nanocapsules instead of PTX, and then TRAIL was conjugated with the pyrene/ F127/P123 silica nanocapsules. The reason for using pyrene was that its absorbances at $338 \mathrm{~nm}$ was proportional to its content. The TRAIL-conjugated pyrene/F127/P123 silica nanocapsules and the pyrene/F127/P123 silica nanocapsules without TRAIL were separated by using Ni-chelating affinity chromatography, and then measured at $338 \mathrm{~nm}$ specific for pyrene. Fig. 1D showed that the absorbances at $338 \mathrm{~nm}$ were 0.978 for TRAILconjugated pyrene/F127/P123 silica nanocapsules and 0.322 for unconjugated nanocapsules. It was calculated that $75.2 \%$ nanocapsules were successfully coupled with TRAIL protien.

\subsection{In vitro cytotoxicity and targeting capability of PFPSNT}

The cytotoxicity of the nanocapsules (NCs) without PTX and TRAIL and anti-tumor cell capacity of PTX, PTX-NCs, TRAIL, TRAIL-NCs and PFPSNT were examined using CCK-8 assays. HepG2 and CaSki cells were respectively used in these assays. As shown in Fig. 4A and B, cell viabilities of HepG2 and CaSki cells were almost $100 \%$ when they were incubated with NCs for $24 \mathrm{~h}$, suggesting that the blank NCs did not show obvious cytotoxicity towards both cancer cells. The relative viabilities of CaSki cells treated respectively with PTX and PTX-NCs showed a comparable decrease with the increase of PTX concentration. In the case of HepG2, trearments by PTX or PTX-NCs also showed a similar result. No significant difference between PTX and PTXNCs treatments indicated again that the NCs did not have observable cytotoxicity. The decrease of relative viabilities might mainly come from the cytotoxicity of PTX. This conclusion was also supported by the half maximal inhibitory concentrations $\left(\mathrm{IC}_{50}\right)$ values of PTX $\left(671.4 \mu \mathrm{g} \mathrm{mL}{ }^{-1}\right.$ for HepG2, $194.4 \mu \mathrm{g} \mathrm{mL} \mathrm{L}^{-1}$

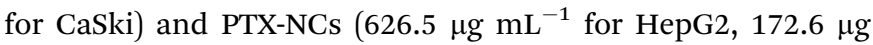
$\mathrm{mL}^{-1}$ for CaSki). $\mathrm{IC}_{50}$ values of TRAIL were $5.514 \mu \mathrm{g} \mathrm{mL} \mathrm{m}^{-1}$ for HepG2 and $1.78 \mu \mathrm{g} \mathrm{mL}{ }^{-1}$ for CaSki. Similarly, $\mathrm{IC}_{50}$ values of TRAIL-NCs were $4.883 \mu \mathrm{g} \mathrm{mL}{ }^{-1}$ for HepG2 and $2.092 \mu \mathrm{g} \mathrm{mL} \mathrm{m}^{-1}$ for CaSki. No significant difference between $\mathrm{IC}_{50}$ values of TRAIL and TRAIL-NCs for both two cell lines, indicating that the bioactivity of TRAIL was not influenced after it was conjugated to silica nanocapsules. In addition, PFPSNT showed the lowest cell viability with the $\mathrm{IC}_{50}$ values of $921.1 \mathrm{ng} \mathrm{mL}{ }^{-1}$ for HepG2 and $236.2 \mathrm{ng} \mathrm{mL}^{-1}$ for CaSki. The PFPSNT had a superior anticancer effect as compared to the PTX-NCs and TRAIL. Those results obtained from in vitro studies demonstrated that the
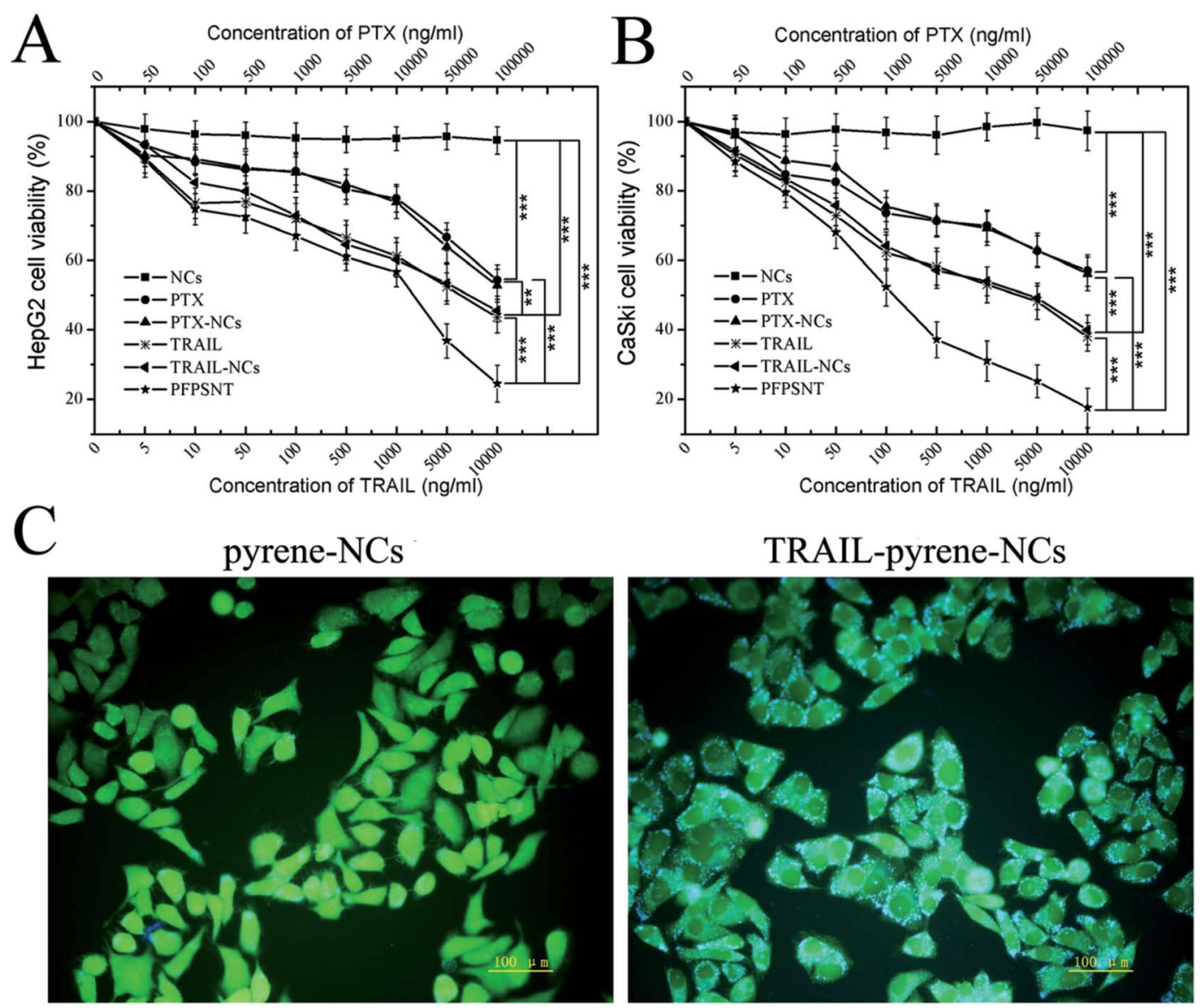

Fig. 4 Synergistic cytotoxicity evaluations. Viabilities of CaSki cells (A) and HepG2 cells (B) treated with NCs, PTX, PTX-NCs, TRAIL, TRAIL-NCs and PFPSNTs; (C) cellular uptake of TRAIL-pyrene-NCs in HepG2. The results from statistic analysis were marked in (A) and (B).*** Means $P$ values of $<0.01 ;{ }^{* *}$ means $P$ values of $<0.05$. 
surface conjugation of the PTX-NCs with TRAIL was indeed able to enhance the efficacy of killing cancer cells.

To test the targeting capability of the PFPSNT, a fluorescent dye pyrene, instead of PTX, was encapsulated into the nanocapsules. Pyrene gives blue emission fluorescence at the $338 \mathrm{~nm}$ excitation. When HepG2 cells were cultured with TRAIL-pyreneNCs or pyrene-NCs without TRAIL conjugation, the cells treated with TRAIL-pyrene-NCs showed a significant increase in the intensity of blue fluorescence compared to those cells treated with pyrene-NCs (Fig. 4C). The cells treated with pyrene-NCs without TRAIL conjugation mainly displayed green fluorescence which came from FDA staining. This result demonstrated that TRAIL indeed had a greatly targeting capacity.

\subsection{Anticancer efficacy of PFPSNT on HepG2 xenografts}

Anticancer efficacy of PFPSNT was investigated in vivo by using the nude mice bearing HepG2 xenografts. The body weights and tumor volumes were measured every two days and summarized in Fig. 5. The mean body weights of mice in all five groups increased slowly with a similar manner (see Fig. 5A). However, the tumor volumes in each group were significantly different (see Fig. 5B). In the control group, five tumors growed rapidly, and the mean volume increased approximately 13 fold in two weeks. The tumor volumes in other three groups treated with PTX, PTX-NCs or TRAIL showed somewhat increase, but much slowly than that for control group. This result indicated that PTX or TRAIL alone was able to suppress tumor growth as expected. In the group of PFPSNT treatment, the PFPSNT showed marked tumor inhibition. After 14 day treatments, the tumors disappeared from two mice in this group, and other three tumors stoped their growth.

After 14 day treatments, the mice were sacrificed and tumors were collected. The photographs of all tumors obtained from five groups were shown in Fig. 5C. The tumor sizes in the control group were significantly larger than those for other four groups. In the PFPSNT-treated group, the sizes of tumors were the smallest, and two tumors disappeared post treatment. Each tumor was weighted, and average weights for each group were shown in Fig. 5D. Average tumor weights were $478 \pm 129 \mathrm{mg}$ for the control group, $172 \pm 56 \mathrm{mg}$ for the PTX-treated group, $116 \pm 47 \mathrm{mg}$ for PTX-NCs-treated group, $88 \pm 25 \mathrm{mg}$ for TRAIL-treated group and $16 \pm 15 \mathrm{mg}$ for the PFPSNT-treated group. These results obtained from in vivo study clearly show that PFPSNT indeed displays high anticancer activity.
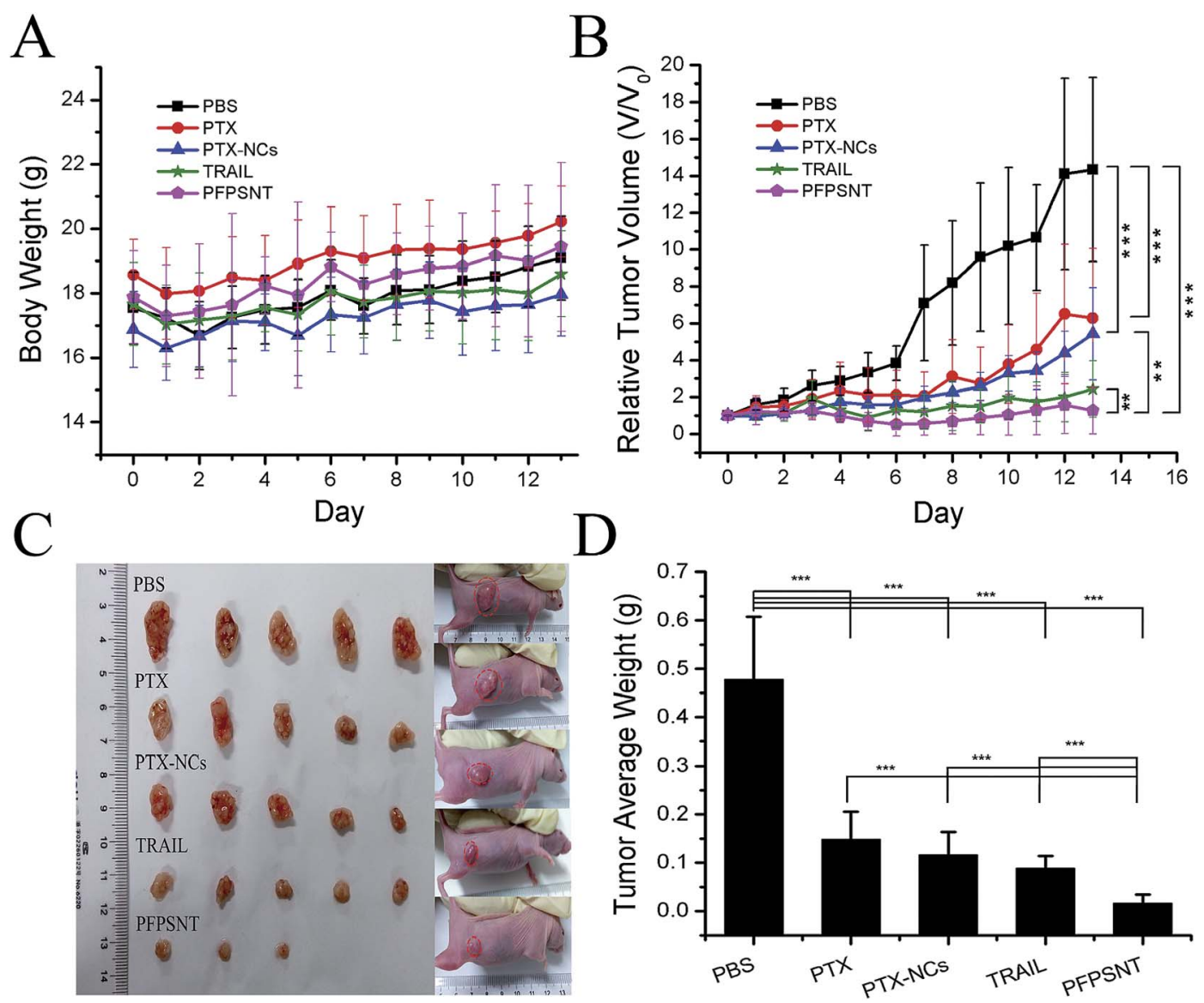

Fig. 5 The body weights (A) and the relative tumor volumes (B) of HepG2 xenografted nu/nu mice treated with PBS, PTX, PTX-NCs, TRAIL and PFPSNT, respectively. (C) Photographs of excised tumors and the mice after 14 day treatments. (D) Mean weights of five tumors in each group after 14 day treatments. ${ }^{* *}$ Presented significant difference $(p<0.01)$, and** means $P$ values of $<0.05$. 


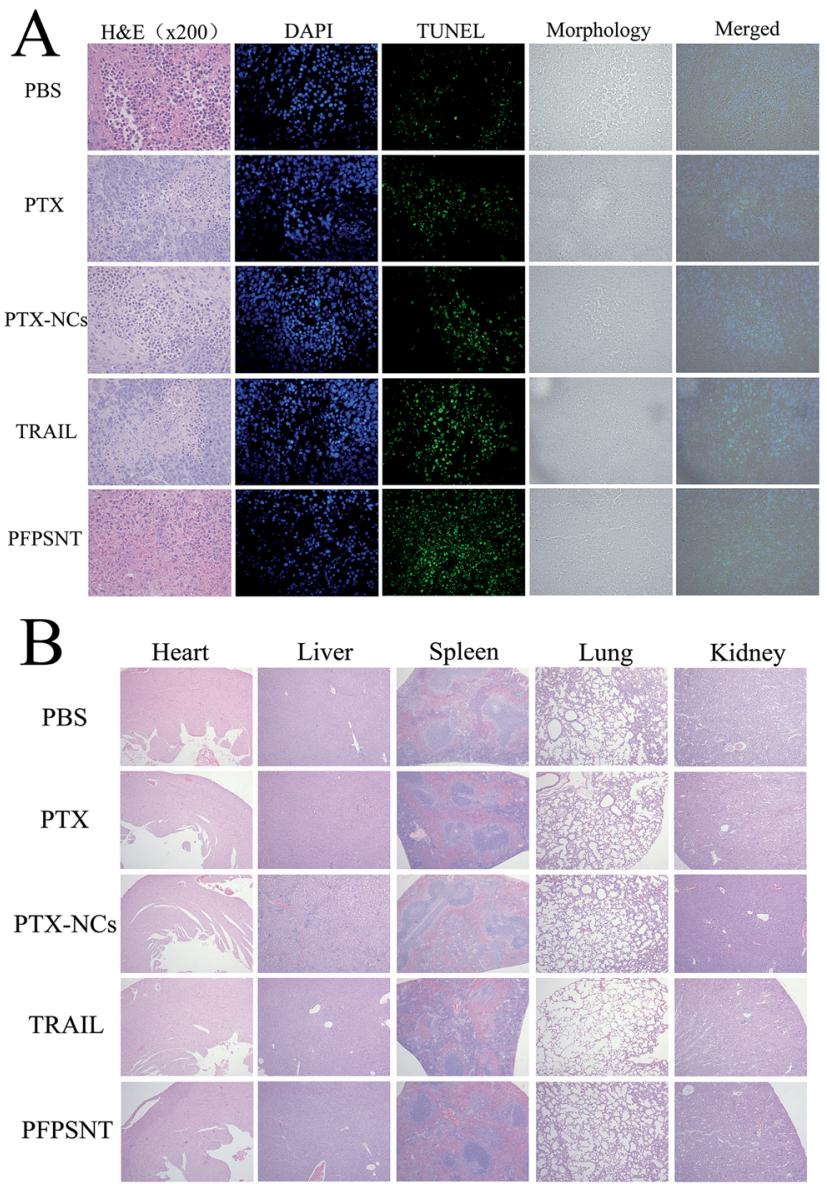

Fig. 6 Histological observations of HepG2 xenografts and major organs of HepG2-xenografted nu/nu mice treated with PTX, PTX-NCs, TRAIL or PFPSNT. (A) The tumor tissues were stained with H\&E, TUNEL and DAPI respectively, and (B) all tissues of mouse organs were stained by H\&E staining.

\subsection{Histological evaluations of HepG2 xenografts and major organs of HepG2 xenografted mice}

Tumor necrosis and apoptosis were visualized by staining with H\&E, TUNEL and DAPI. As shown in Fig. 6A, there were obvious tumor cells with little red cytoplasm and big purple nucleus, while vascular in tumor also could be found. Tumor necrosis area of H\&E stained tumor tissues were statistical analysis by Image-pro Plus and SPSS. The mean tumor necrosis area of PFPSNT, TRAIL, PTX-NCs, PTX and control groups were $38.8 \%, 31.1 \%, 23.9 \%, 17.2 \%$ and $9.4 \%$. The tumor tissues from five groups were stained with TUNEL and observed under fluorescence microscope. In this experiment, apoptotic cells displayed green fluorescence. As shown in Fig. 6A, the tumor tissue treated with PFPSNT showed much stronger green fluorescence as compared to other four groups, indicating that the combination of TRAIL and PTX indeed induced cell apoptosis, which was more than those treated with single therapeutic approach alone. DAPI staining showed a smaller amount of nucleus presented in the tumor tissues of the PFPSNT-treated group, indicating that more cell necrosis happened in this group. Tumor tissues photos viewed in the bright field and merged picture were also shown in Fig. 6A.

In vivo toxicity was also examined via $\mathrm{H} \& \mathrm{E}$ staining of major organs of the mice bearing HepG2 xenografts used in this study, including heart, liver, spleen, lung and kidney. As shown in Fig. 6B, no obvious lesion or inflammation was found in all histological samples collected from five groups. These results showed that the nano material and TRAIL protein used in this study had a good biocompatibility, and could be used as drug carriers for further clinical treatment.

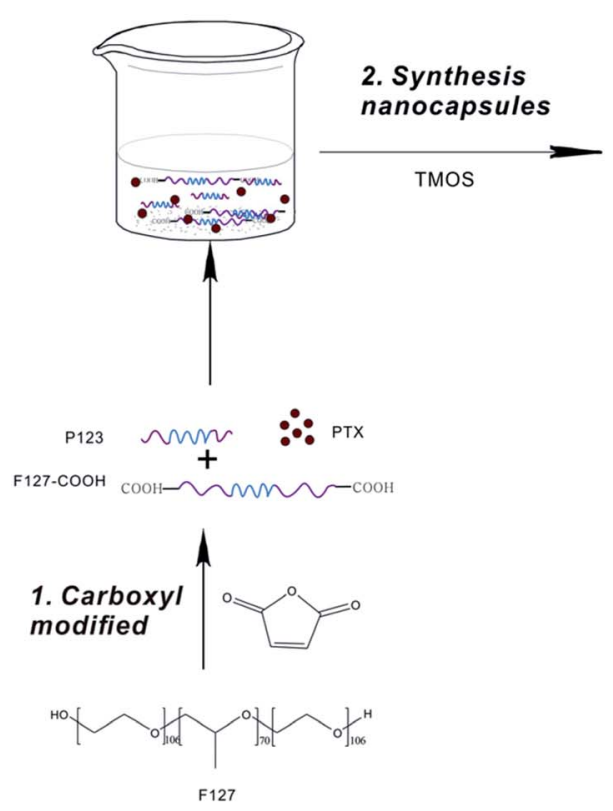

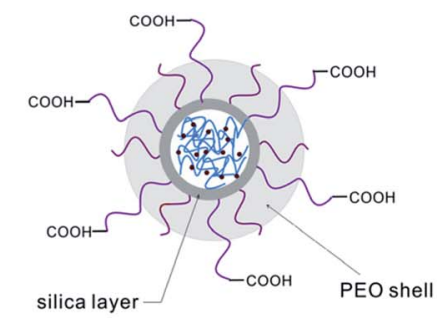
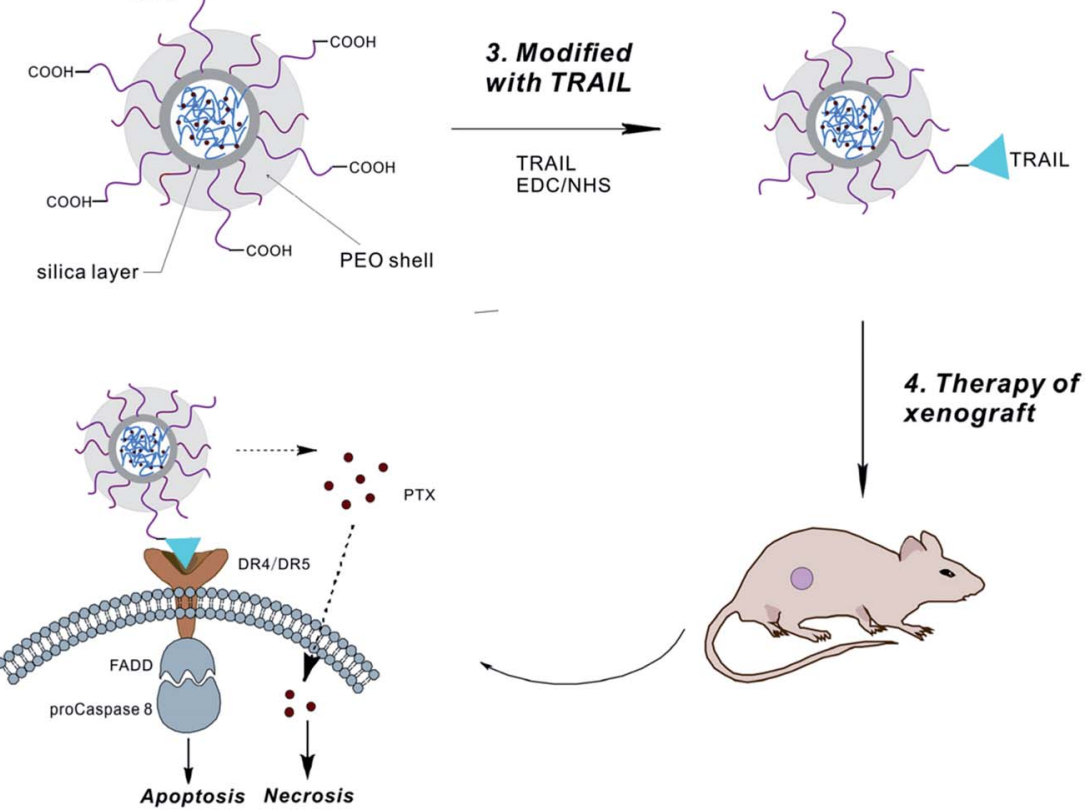

Fig. 7 A schematic presenting the synthetical route of the novel nano-drug PFPSNT and its possible anticancer mechnism. 


\section{Conclusion}

In this study, we describe a novel strategy to develop an anticancer drug for targeting cancer therapy, in which PTX-NCs was made firstly and then conjugated with TRAIL to form a novel nano-drug PFPSNT. We expected that TRAIL linked to the carboxylic groups of the F127 on the surface of PFPSNT acted as a direct anticancer reagent and a delivery vector targeting DR4 and DR5 death receptors of cancer cells. Meanwhile, PTX embedded in pluronic F127/P123 silica nanocapsules acted as another anticancer reagent and performed a sustained release in order to reduce its side effects during cancer therapy. Fig. 7 presented the technical route for synthesizing PFPSNT and a possible anticancer mechanism. At first, PFPSNT targets cancer cells via interactions of TRAIL with death receptors DR4 and DR5, and then TRAIL induces apoptosis of tumor cells. Meanwhile, PTX released from PFPSNT goes directly into tumor cells to cause cell necrosis. As expected, the pluronic F127/P123 silica nanocapsules prepared in this study were spherical nanoparticles with a size of $24 \mathrm{~nm}$, and more stabile than the F127/P123 nanomicelles. Polymeric F127/P123 silica nanocapsule indeed enhanced PTX solubility, and sustained PTX release. In both in vitro and in vivo studies, the PFPSNT made in this study showed a strong capability for killing HepG2 and CaSki cells and restraining the growth of HepG2 xenografts without impairment of host organs. As a result, PFPSNT could be considered as a promising drug to treat complex tumors in targeting cancer therapy.

\section{Ethical statement}

Animals, used in this study, were maintained in accordance with the guidelines of the national standard of China for laboratory animal-requirements of environment and housing facilities (GB 14925-2010), and approved by the institutional committee of the Laboratory Animal Center, Huazhong University of Science and Technology.

\section{Acknowledgements}

This work was supported by the grant (51303046) founded by National Natural Science Foundation of China, and the science and technology project of Finance Department of Hubei Province. The authors also would like to thank the Laboratory Animal Center, Huazhong University of Science and Technology for its kind assistances and guidelines of animal experiments.

\section{References}

1 G. Pan, K. O'Rourke, A. M. Chinnaiyan, et al., The receptor for the cytotoxic ligand TRAIL, Science, 1997, 276, 111-113.

2 R. M. Pitti, S. A. Marsters, S. Ruppert, et al., Induction of apoptosis by Apo-2 ligand, a new member of the tumor necrosis factor cytokine family, J. Biol. Chem., 1996, 271, 12687-12690.
3 J. J. Song and Y. J. Lee, Differential cleavage of Mst1 by caspase-7/-3 is responsible for TRAIL-induced activation of the MAPK superfamily, Cell. Signalling, 2008, 20, 892-906.

4 R. S. Herbst, S. G. Eckhardt, R. Kurzrock, S. Ebbinghaus, P. J. O'Dwyer, M. S. Gordon, W. Novotny, M. A. Goldwasser, T. M. Tohnya, B. L. Lum, et al., Phase I dose-escalation study of recombinant human Apo2L/TRAIL, a dual proapoptotic receptor agonist, in patients with advanced cancer, J. Clin. Oncol., 2010, 28, 2839-2846.

5 J. C. Soria, E. Smit, D. Khayat, B. Besse, X. Yang, C. P. Hsu, D. Reese, J. Wiezorek and F. Blackhall, Phase 1b study of dulanermin (recombinant human Apo2L/TRAIL) in combination with paclitaxel, carboplatin, and bevacizumab in patients with advanced non-squamous non-small-cell lung cancer, J. Clin. Oncol., 2010, 28, 1527-1533.

$6 \mathrm{~J}$. Wiezorek, P. Holland and J. Graves, Death receptor agonists as a targeted therapy for cancer, Clin. Cancer Res., 2010, 16, 1701-1708.

7 L. Zhang and B. Fang, Mechanisms of resistance to TRAILinduced apoptosis in cancer, Cancer Gene Ther., 2005, 12, 228-237.

8 C. S. Mitsiades, S. P. Treon, N. Mitsiades, et al., TRAIL/Apo2L ligand selectively induces apoptosis and overcomes drug resistance in multiple myeloma: therapeutic applications, Blood, 2001, 98, 795-804.

9 S. Fulda, W. Wick, M. Weller, et al., Smac agonists sensitize for Apo2L/TRAIL- or anticancer drug-induced apoptosis and induce regression of malignant glioma in vivo, Nat. Med., 2002, 8, 808-815.

10 M. Garofalo, G. D. Leva, G. Romano, et al., miR-221\&222 Regulate TRAIL Resistance and Enhance Tumorigenicity through PTEN and TIMP3 Downregulation, Cancer Cell, 2009, 16, 498-509.

11 U. M. Nazim, J. K. Jeong, J. W. Seol, J. Hur, S. K. Eo, J. H. Lee and S. Y. Park, Inhibition of the autophagy flux by gingerol enhances TRAIL-induced tumor cell death, Oncol. Rep., 2015, 33, 2331-2336.

12 S. Ray and A. Almasan, Apoptosis Induction in Prostate Cancer Cells and Xenografts by Combined Treatment with Apo2 Ligand/Tumor Necrosis Factor-related Apoptosisinducing Ligand and CPT-11, Cancer Res., 2003, 63, 47134723.

13 I. Kim, H. J. Byeon, T. H. Kim, et al., Doxorubicin-loaded porous PLGA microparticles with surface attached TRAIL for the inhalation treatment of metastatic lung cancer, Biomaterials, 2013, 34, 6444-6453.

14 S. Bae, K. Ma, T. H. Kim, et al., Doxorubicin-loaded human serum albumin nanoparticles surface-modified with TNFrelated apoptosis-inducing ligand and transferrin for targeting multiple tumor types, Biomaterials, 2012, 33, 1536-1546.

15 E. Pasquier, M. Carré, B. Pourroy, L. Camoin, O. Rebaï, C. Briand and D. Braguer, Antiangiogenic activity of paclitaxel is associated with its cytostatic effect, mediated by the initiation but not completion of a mitochondrial apoptotic signaling pathway, Mol. Cancer Ther., 2004, 3, 1301-1310. 
16 L. Li, X. Wen, Z. Bu, X. Cheng, X. Xing, X. Wang, L. Zhang, T. Guo, H. Du, B. Fan and J. Ji, Paclitaxel enhances tumoricidal potential of TRAIL via inhibition of MAPK in resistant gastric cancer cells, Oncol. Rep., 2016, 35, 30093027.

17 J. Gong, D. Yang, S. Kohanim, R. Humphreys, L. Broemeling and R. Kurzrock, Novel in vivo imaging shows up-regulation of death receptors by paclitaxel and correlates with enhanced antitumor effects of receptor agonist antibodies, Mol. Cancer Ther., 2006, 5, 2991-3000.

18 T. B. Hunter, N. J. Manimala, K. A. Luddy, T. Catlin and S. J. Antonia, Paclitaxel and TRAIL synergize to kill paclitaxel-resistant small cell lung cancer cells through a caspase-independent mechanism mediated through AIF, Anticancer Res., 2011, 31, 3193-3204.

19 R. Nimmanapalli, C. L. Perkins, M. Orlando, E. O'Bryan, D. Nguyen and K. N. Bhalla, Pretreatment with paclitaxel enhances apo-2 ligand/tumor necrosis factor-related apoptosis-inducing ligand induced apoptosis of prostate cancer cells by inducing death receptors 4 and 5 protein levels, Cancer Res., 2001, 61, 759-763.

20 E. K. Rowinsky and R. C. Donehower, Paclitaxel (taxol), N. Engl. J. Med., 1995, 332, 1004-1014.

21 J. D. Adams, K. P. Flora, B. R. Goldspiel, et al., Taxol: a history of pharmaceutical development and current pharmaceutical concerns, JNCI Monogr., 1993, 15, 141-147.

22 H. Gelderblom, J. Verweij, K. Nooter, et al., Cremophor EL: the drawbacks and advantages of vehicle selection for drug formulation, Eur. J. Cancer, 2001, 37, 1590-1598.

23 H. Xin, L. Chen, J. Gu, et al., Enhanced anti-glioblastoma efficacy by PTX-loaded PEGylated poly( $\varepsilon$-caprolactone) nanoparticles: in vitro and in vivo evaluation, Int. J. Pharm., 2010, 402, 238-247.

24 Y. Saito, Nanoparticles and filled nanocapsules, Carbon, 1995, 33, 979-988.

25 C. E. Mora-Huertas, H. Fessi and A. Elaissari, Polymer-based nanocapsules for drug delivery, Int. J. Pharm., 2010, 385, 113-142.

26 P. Couvreur, G. Barratt, E. Fattal, et al., Nanocapsules Technology: A review, Crit. Rev. Ther. Drug Carrier Syst., 2002, 19, 99-134.

27 A. L. Savian, D. Rodrigues, J. Weber, et al., Dithranol-loaded lipid-core nanocapsules improve the photostability and reduce the in vitro irritation potential of this drug, Mater. Sci. Eng., C, 2015, 46, 69-76.

28 R. H. Staff, M. Gallei, M. Mazurowski, et al., Patchy nanocapsules of poly(vinylferrocene)-based block copolymers for redox-responsive release, ACS Nano, 2012, 6, 9042-9049.

29 F. Chécot, J. Rodríguez-Hernández, Y. Gnanou, et al., pHresponsive micelles and vesicles nanocapsules based on polypeptide diblock copolymers, Biomol. Eng., 2007, 24, 8185.

30 S. Peltier, J. M. Oger, F. Lagarce, et al., Enhanced Oral Paclitaxel Bioavailability After Administration of PaclitaxelLoaded Lipid Nanocapsules, Pharm. Res., 2006, 23, 12431250 .
31 D. Ren, F. Kratz and S. W. Wang, Protein nanocapsules containing doxorubicin as a pH-responsive delivery system, Small, 2011, 7, 1051-1060.

32 V. Chupin, A. I. P. M. De Kroon and B. De Kuijff, Lipid organization in cisplatin nanocapsules: lipid-coated aggregates of cisplatin with high cytotoxicity, Cell. Mol. Biol. Lett., 2005, 10, 81-84.

33 Y. Zhang, B. Y. Hsu, C. Ren, X. Li and J. Wang, Silica-based nanocapsules: synthesis, structure control and biomedical applications, Chem. Soc. Rev., 2015, 44, 315.

34 K. Tatematsu, M. Iijima, N. Yoshimoto, et al., Bionanocapsules displaying various immunoglobulins as an active targeting-based drug delivery system, Acta Biomater., 2016, 35, 238-247.

35 X. G. Wang, T. Jandl, E. Dadachova, et al., Effect of naive and radiolabeled rhTRAIL on the cervical cancer xenografts in mice, Ther. Delivery, 2014, 5, 139-147.

36 S. J. Huang, S. L. Sun, T. H. Feng, et al., Folate-mediated chondroitin sulfate-Pluronic 127 nanogels as a drug carrier, Eur. J. Pharm. Sci., 2009, 38, 64-73.

37 F. Szoka Jr and D. Papahadjopoulos, Comparative Properties and Methods of Preparation of Lipid Vesicles (Liposomes), Annu. Rev. Biophys. Bioeng., 1980, 9, 467-508.

38 Y. Zhang, M. Wang, Y. Zheng, et al., PEOlated Micelle/Silica as Dual-Layer Protection of Quantum Dots for Stable and Targeted Bioimaging, Chem. Mater., 2013, 25, 2976-2985.

39 T. Zhao, X. Liu, Y. Li, et al., Fluorescence and drug loading properties of $\mathrm{ZnSe}: \mathrm{Mn} / \mathrm{ZnS}-\mathrm{Paclitaxel} / \mathrm{SiO}_{2}$ nanocapsules templated by F127 micelles, J. Colloid Interface Sci., 2016, 490, 436-443.

40 W. Zhang, Y. Shi, Y. Chen, et al., Multifunctional Pluronic P123/F127 mixed polymeric micelles loaded with paclitaxel for the treatment of multidrug resistant tumors, Biomaterials, 2011, 32, 2894-2906.

41 A. Andersen, D. J. Warren, P. F. Brunsvig, et al., High sensitivity assays for docetaxel and paclitaxel in plasma using solid-phase extraction and high-performance liquid chromatography with UV detection, BMC Clin. Pharmacol., 2006, 6, 1-10.

42 M. Tsai, Z. Lu, M. G. Wientjes, et al., Paclitaxel-loaded polymeric microparticles: Quantitative relationships between in vitro, drug release rate and in vivo, pharmacodynamics, J. Controlled Release, 2013, 172, 737744.

43 J. Hong, D. Xu, P. Gong, et al., Covalent binding of $\alpha$ chymotrypsin on the magnetic nanogels covered by amino groups, J. Mol. Catal. B: Enzym., 2007, 45, 84-90.

44 P. Demaerel, H\&E Staining, Encyclopedia of Diagnostic Imaging, Springer, Berlin, Heidelberg, 2008.

45 F. Otto, DAPI staining of fixed cells for high-resolution flow cytometry of nuclear DNA, Methods Cell Biol., 1990, 33, 105110.

46 S. W. Perry, L. G. Epstein and H. A. Gelbard, Simultaneous in situ detection of apoptosis and necrosis in monolayer cultures by TUNEL and trypan blue staining, BioTechniques, 1997, 22, 1102-1106. 
47 R. R. Wilcox, Two-way ANOVA, Applying Contemporary Statistical Techniques, 2003.

48 W. Zhang, J. Hao, Y. Shi, et al., Paclitaxel-loaded Pluronic P123/F127 mixed polymeric micelles: formulation, optimization and in vitro characterization, Int. J. Pharm., 2009, 376, 176-185.

49 H. Tan, N. S. Liu, B. He, et al., Facile synthesis of hybrid silica nanocapsules by interfacial templating condensation and their application in fluorescence imaging, Chem. Commun., 2009, 6240-6242.
50 B. Y. W. Hsu, C. Teh, H. Tan, et al., PEO surface-decorated silica nanocapsules and their application in in vivo imaging of zebrafish, RSC Adv., 2012, 2, 12392-12399.

51 N. Rapoport, Stabilization and activation of Pluronic micelles for tumor-targeted drug delivery, Colloids Surf., B, 1999, 16, 93-111.

52 R. P. Shringi, PyMol Software for 3D Visualization of Aligned Molecules, Biomaterials, 2005, 26, 63-72. 